\title{
Transgastric Pancreatic Necrosectomy: How I Do It
}

\author{
Nicholas J. Zyromski, MD \\ Attila Nakeeb, MD \\ Michael G. House, MD \\ Andrea L. Jester, MD
}

Address correspondence to:

Nicholas J. Zyromski, MD

Associate Professor,

Department of Surgery

Indiana University School of Medicine

545 Barnhill Drive, EH 519

Indianapolis, IN 46202

Phone 317-274-5012

Fax 317-274-0241

Email: nzyromsk@iupui.edu

This is the author's manuscript of the article published in final edited form as:

Zyromski, N. J., Nakeeb, A., House, M. G., \& Jester, A. L. (2015). Transgastric Pancreatic Necrosectomy: How I Do It. Journal of Gastrointestinal Surgery, 20(2), 445-449. http://doi.org/10.1007/s11605-015-3058-y 


\section{ABSTRACT}

Necrotizing pancreatitis is a serious medical problem that often requires intervention to debride necrotic pancreatic and peripancreatic tissue. Recently, minimally invasive approaches have been applied to pancreatic necrosectomy. The purpose of this report is to review the history of transgastric pancreatic debridement, identify appropriate patient selection criteria, and highlight technical "pearls." We present this subject matter in the context of our own clinical experience, with a primary focus on a "How I Do It" type of technical description.

\section{BACKGROUND}

Acute pancreatitis is a common medical problem effecting over one quarter million patients yearly in the United States (1). Among these patients, $15-20 \%$ will suffer a severe episode of pancreatitis with variable necrosis of the pancreatic and peripancreatic soft tissue. Patients with necrotizing pancreatitis often require intervention to treat infected necrosis or symptomatic necrosis. The natural history of pancreatic and peripancreatic necrosis is shown in Figure 1.

Recently, minimally invasive approaches have been applied to treatment of pancreatic necrosis. These approaches include percutaneous drainage, endoscopic drainage (and debridement), a combination of the percutaneous and endoscopic approaches, laparoscopy, and retroperitoneal approaches (videoscopic assisted retroperitoneal debridement [VARD] - and sinus tract necrosectomy). (2-9)

It is important to recognize that necrotizing pancreatitis is an extremely heterogeneous disease; as such, no one specific technique is suitable to treat all 
patients. Surgeons who care for necrotizing pancreatitis patients should be prepared to provide long-term care and follow up. Ideally, these patients are approached in the context of a multidisciplinary team including gastroenterologists, interventional radiologists, and pancreatic surgeons, with additional support from intensive care doctors, nutritionists, and physical therapists.

\section{HISTORY OF TRANSGASTRIC DEBRIDEMENT}

Barron and colleagues reported the endoscopic transgastric approach in 1996

(3). Several other groups have subsequently explored endoscopic debridement. (4) The first laparoscopic transgastric debridement was reported in 2002. (10) In 2008, a novel laparoendoscopic rendez-vous approach was reported by the Freiburg group, (8) This report consisted of 6 patients. More recently, 4 reports of conventional operative transgastric debridement have been published. A series of 7 patients from Denmark was reported in 2007, and a series of 10 patients were treated by the transgastric approach by the pancreatic surgery group at the University of Calgary $(11,12)$. The well-established Glasgow group recently published the most robust report of transgastric approach to date. (13) This series documented outcomes of 44 patients approached through the back wall of the stomach, 8 open and 36 laparoscopic. Most recently, the Stanford group reported their experience with 21 patients; in the Stanford experience, no intervention has been necessary at very short follow up. (14) The table summarizes contemporary reports of transgastric surgical necrosectomy.

All of these reports recognize the potential for hemorrhage while transgressing the gastric wall. This potential problem is not surprising as many patients with lesser sac pancreatic necrosis have splenic vein thrombosis with left sided (sinistral) portal 
hypertension. In addition, long-term follow up of patients treated by transgastric necrosectomy is modest. The true incidence of long-term complications such as reaccumulation of retroperitoneal fluid or left sided acute pancreatitis remains to be defined completely.

\section{TRANSGASTRIC ADVANTAGES AND PATIENT SELECTION}

Transgastric necrosectomy is attractive as it allows thorough debridement of retroperitoneal necrosis at one setting. This is in contrast to the endoscopic approach, which typically requires multiple interventions at separate settings. Combining transgastric debridement with what is essentially cysto-gastrostomy also offers the potential for durable internal drainage of a disconnected pancreatic tail- the so-called "el Diablo" because of its devilish nature. In a follow up over a 6-year period we have found several patients with recurrent left sided pancreatitis and/or recurrent retroperitoneal collections; therefore, we typically counsel patients about the potential for recurrent problems after transgastric debridement. Finally, operative approach allows cholecystectomy (with cholangiography) to be performed at the same setting for patients with biliary acute pancreatitis.

The ideal patient to select for transgastric necrosectomy has necrosis confined to the lesser sac. Figure 2 illustrates typical patterns of necrosis in necrotizing pancreatitis. Patients with necrosis extending down the left paracolic gutter may be better approached with VARD. Patients whose necrosis extends down the root of the small bowel mesentery are more problematic. We have treated at least 2 patients with this pattern of necrosis by transgastric necrosectomy with an additional large closed 
suction drain placed down the space of the mesenteric root. The drain is subsequently withdrawn slowly, starting 3-4 weeks after operation. Patients with necrosis that involves the pancreatic head and extends down the right paracolic gutter are extremely challenging regardless of approach. In this setting, clinician and patient should be prepared for long treatment course with a potential for duodenal and/or bile duct strictures.

It is important to remember lessons from open necrosectomy regarding timing of definitive debridement. Timing of definitive intervention should be delayed at least 1 month from the initial insult to allow consolidation of the necrosis in the lesser sac. This consolidation greatly simplifies debridement and makes the procedure much less hazardous with regard to potential for hemorrhage from the retroperitoneum.

All necrotizing pancreatitis patients have some degree of malnutrition; alimenting the gut is important preoperatively, and gastrojejunostomy feeding tube at the time of transgastric debridement should be considered. Our preference is to place a gastrojejunostomy feeding tube as many patients have gastric ileus from the lesser sac inflammatory condition; they may decompress ("vent") the stomach by the "G"port while feeding the small bowel distal to Treitz' ligament through the "J" port. Early infection should be managed by percutaneous drainage to temporize the immediate clinical situation. It is not clear whether percutaneous drain placement is a contraindication to subsequent transgastric debridement. 
TRANSGASTRIC DEBRIDEMENT - SURGICAL TECHNIQUE

Transgastric debridement may be approached laparoscopically or through a short upper midline incision. Laparoscopic ports are placed similar to other foregut operations (Figure 3). The epigastric port allows the operator to drive the camera directly into the necrosis cavity (Figure 4). Intraoperative ultrasound is essential to help define the necrotic collection location, extent, and compositon, particularly when the necrosis is predominately solid with a minimal fluid component. (Figure 5) Anterior gastrostomy is created between stay sutures using electrocautery or the ultrasonic scalpel. It is worth reiterating the potential for major hemorrhage from the gastric wall in the setting of left sided portal hypertension. In this situation the surgical approach clearly offers an advantage over endoscopic approach in facilitating hemorrhage control. Placing the ultrasound transducer directly into the stomach and visualizing the retrogastric collection through the posterior wall of the stomach is helpful in positioning the posterior gastrostomy. Posterior gastrostomy is created between stay sutures;

these sutures are particularly helpful during the laparoscopic approach manipulating the gastrostomy into the operative field. Cultures of the retrogastric fluid and necrosis are routine as $25-30 \%$ of pancreatic collections considered sterile actually harbor subclinical infection. The posterior gastrostomy/cysto-gastrostomy is enlarged with electrocautery, ultrasonic shears, or by firing an endovascular staple load between the cyst wall and the stomach. Most times this clinical situation is clearly acute pancreatitis; however, biopsy of the cyst wall may be sent to prove absence of epithelialization, i.e. neoplastic cyst.

Debridement of the necrotic material is undertaken gently either with "the educated finger" in the open approach or with a blunt grasper in the laparoscopic 
approach (Figure 6). It is critical not to debride solid tissue that is not loose and easily dislodged from the retroperitoneum. Debridement of immature necrosis may result in catastrophic hemorrhage. Visualization to the right of the superior mesenteric vein and down the root of the small bowel mesentery is challenging laparoscopically; concomitant endoscopy may be helpful in these situations. We have found that vigorous irrigation of the necrosis cavity is useful in dislodging small particulate solid matter. The necrosis cavity may be packed with a gauze sponge which typically tamponades small oozing vessels in the retroperitoneum. Necrotic material may be placed into an endocatch pouch device for retrieval from the abdomen; alternately, necrosis may simply be left within the stomach lumen, where it will pass through the alimentary tract. After satisfactory debridement has been achieved, cysto-gastrostomy is secured either with monofilament nonabsorbable suture or with a linear endovascular stapler. If a gastrojejunostomy feeding tube is indicated, it is placed through a separate purse string suture in the anterior stomach at this time; the anterior gastrostomy aids visualization directing this feeding tube beyond the pylorus. Anterior gastrostomy is closed either with a stapler or more commonly with 2 layers of suture, as the stomach is often thickened and not ideal for a stapled closure (Figure 7). Cholecystectomy may be addressed at this point if indicated. Some operators may prefer to perform cholecystectomy prior to gastrotomy and debridement. POSTOPERATIVE CARE AND LONG TERM OUTCOMES

With sterile necrosis, antibiotics are not routinely administered beyond the perioperative period. Infected necrosis should be treated with a 7-10 day antibiotic course tailored to cover cultured organisms. Longer antibiotic courses should be 
considered to treat resistant bacteria, which are extremely challenging to clear from the retroperitoneal space. Diet is advanced as tolerated, understanding that patients with long standing lesser sac inflammatory collections may have a protracted gastric ileus. Venous thromboembolic events are extremely common in necrotizing pancreatitis; therefore, pharmacologic prophylaxis is prudent. Our practice is to use low molecular weight heparin preparation subcutaneous injections. Finally, patients should be counseled about the potential for recurrent left sided pancreatitis and/or retroperitoneal collections. Over the past few years, we have found approximately $15 \%-20 \%$ of necrotizing pancreatitis patients to be suitable candidates for transgastric debridement.

\section{CONCLUSION}

Surgical transgastric pancreatic necrosectomy is an important treatment option for select patients with necrotizing pancreatitis. This approach is particularly useful when applied to patients with solid necrosis localized in the lesser sac, including those with a disconnected pancreatic tail. The potential for major hemorrhage from the gastric wall should be appreciated. Long-term follow up for patients undergoing this procedure is evolving; and should be mandatory.

\section{ACKNOWLEDGEMENT}

The author thanks Shella Stephens for excellent administrative support preparing the manuscript. 
TABLE: Series of transgastric necrosectomy

\begin{tabular}{llccc} 
Year & Author (reference) & $\mathrm{n}$ & $\begin{array}{c}\text { Follow up } \\
\text { (months) }\end{array}$ & $\begin{array}{c}\text { Late Complication/ } \\
\text { Re-intervention }\end{array}$ \\
\hline 2007 & Ainsworth (11) & 7 & 3 & $3(43 \%)$ \\
2008 & Fischer (8) & 6 & 14 & $2(33 \%)$ \\
2010 & Munene (12) & 10 & 18 & $2(20 \%)$ \\
2014 & Gibson (13) & 44 & 30 & $1(2 \%)$ \\
2014 & Worhunsky (14) & 21 & 11 & $0(0 \%)$
\end{tabular}




\section{FIGURE LEGENDS}

Figure 1: Natural history of pancreatic and peripancreatic necrosis. A small percentage of patients will resolve necrosis spontaneously. Infected and persistent, symptomatic necrosis typically demand intervention.

Figure 2: Typical patterns of necrosis. Necrosis confined to the lesser sac (left figure) is ideal for the transgastric approach. Necrosis extending to the left paracolic gutter (middle figure) may be better off treated with VARD. Necrosis involving the pancreatic head, right paracolic gutter, or small bowel mesenteric root (right figure) is quite challenging to manage, and may be best approached by open transabdominal debridement.

Figure 3: Typical port placement for laparoscopic transgastric necrosectomy. $12 \mathrm{~mm}$ ports are commonly placed at the umbilicus and in the right lower abdominal positions to accommodate ultrasound probe and stapling devices.

Figure 4: Laparoscopic view of the necrosis cavity through the posterior gastric wall after thorough debridement: note disconnected pancreatic tail (long arrow) and splenic artery (short arrow).

Figure 5: Intraoperative ultrasound guidance is essential, particularly in laparoscopy.

Figure 6: Necrosis should be debrided bluntly, and may be placed into a bag or simply left in the gastric lumen.

Figure 7: The anterior gastrotomy is closed with staples or suture. 


\section{REFRENCES}

1. Fagenholz PJ, Fernandez-del Castillo C, Harris NS, Pelletier AJ, Camargo CA, Jr. Direct medical costs of acute pancreatitis hospitalizations in the United States. Pancreas 2007;35:302-7.

2. Freeny PC, Hauptmann E, Althaus SJ, Traverso LW, Sinanan M. Percutaneous CTguided catheter drainage of infected acute necrotizing pancreatitis: techniques and results. AJR American journal of roentgenology 1998;170:969-75.

3. Baron TH, Thaggard WG, Morgan DE, Stanley RJ. Endoscopic therapy for organized pancreatic necrosis. Gastroenterology 1996;111:755-64.

4. Gardner TB, Coelho-Prabhu N, Gordon SR, Gelrud A, Maple JT, Papachristou GI et al. Direct endoscopic necrosectomy for the treatment of walled-off pancreatic necrosis: results from a multicenter U.S. series. Gastrointest endoscopy 2011;73:718-26.

5. Horvath K, Freeny P, Escallon J, Heagerty P, Comstock B, Glickerman DJ, Bulger E, et al. Safety and efficacy of video-assisted retroperitoneal debridement for infected pancreatic collections: a multicenter, prospective, single-arm phase 2 study. Arch surg 2010;145:817-25.

6. Raraty MG, Halloran CM, Dodd S, Ghaneh P, Connor S, Evans J, Sutton R, Neoptolemos JP. Minimal access retroperitoneal pancreatic necrosectomy: improvement in morbidity and mortality with a less invasive approach. Ann Surg 2010;251:787-93.

7. van Santvoort HC, Besselink MG, Bakker OJ, Hofker HS, Boermeester MA, Dejong CH, van Goor $\mathrm{H}$, et al. A step-up approach or open necrosectomy for necrotizing pancreatitis. NEJM 2010;362:1491-502.

8. Fischer A, Schrag HJ, Keck T, Hopt UT, Utzolino S. Debridement and drainage of walled-off pancreatic necrosis by a novel laparoendoscopic rendezvous maneuver: experience with 6 cases. Gastrointest Endoscopy 2008;67:871-8.

9. Parekh D. Laparoscopic-assisted pancreatic necrosectomy: A new surgical option for treatment of severe necrotizing pancreatitis. Arch Surg 2006;141:895-902; discussion -3.

10. Ammori BJ. Laparoscopic transgastric pancreatic necrosectomy for infected pancreatic necrosis. Surg Endoscopy 2002;16:1362.

11. Ainsworth AP, Nielsen HO, Mortensen MB. [Transgastric necrosectomy by open surgery in necrotising acute pancreatitis]. Ugeskrift for laeger 2007;169:126-8.

12. Munene G, Dixon E, Sutherland F. Open transgastric debridement and internal drainage of symptomatic non-infected walled-off pancreatic necrosis. HPB 2011;13:234-9.

13. Gibson SC, Robertson BF, Dickson EJ, McKay CJ, Carter CR. "Step port" laparoscopic cystgastrostomy for the management of organized solid predominant post-acute fluid collections after severe acute pancreatitis. HPB 2014; 16(2): 170-6. 
14. Worhunsky DJ, Qadan M, Dua MM, et al. Laparoscopic transgastric necrosectomy for the management of pancreatic necrosis. J Am Coll Surg 2014; 219 (4): 735-43 
Figure 1:

\section{AP - NATURAL HISTORY}

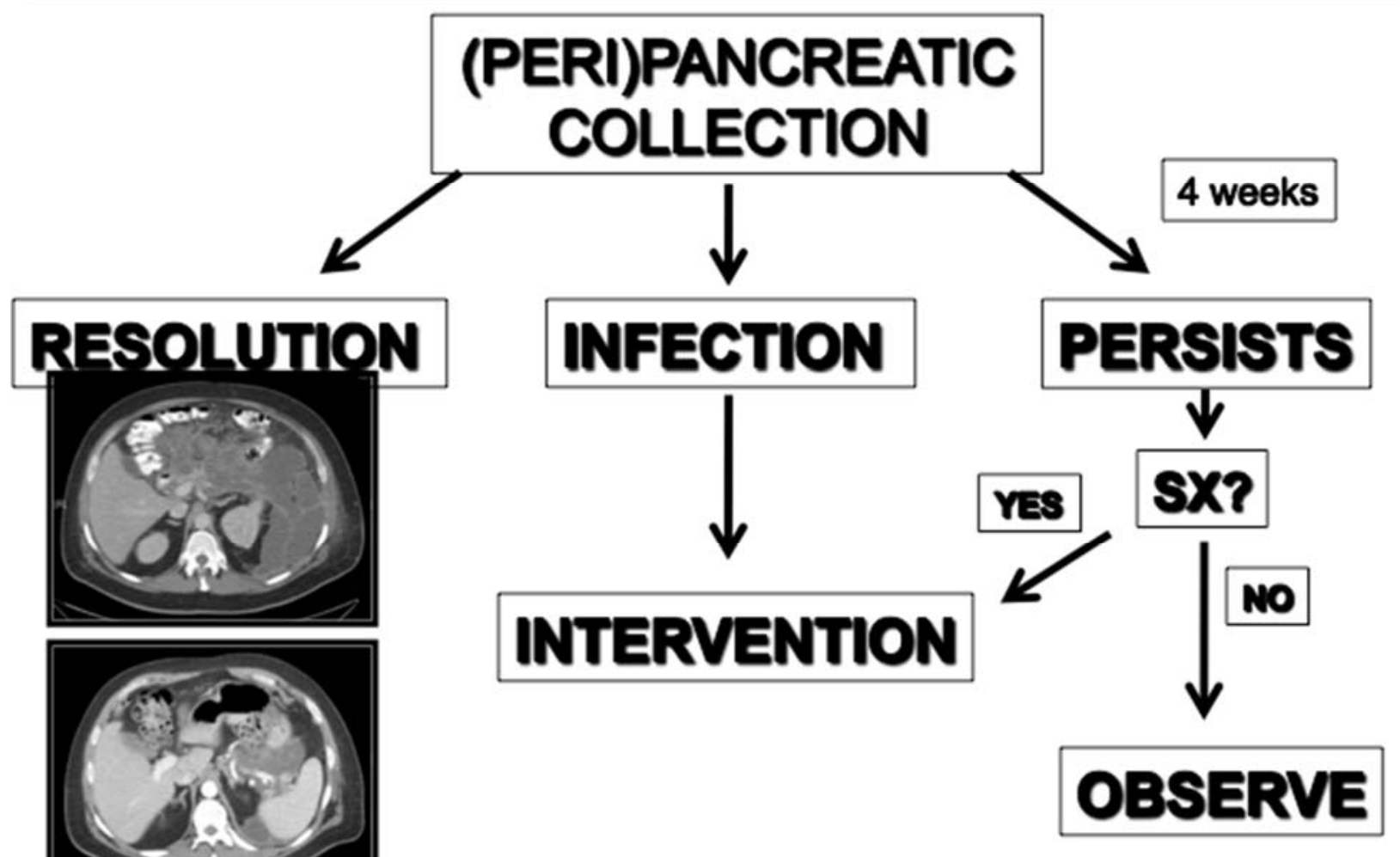


Figure 2:

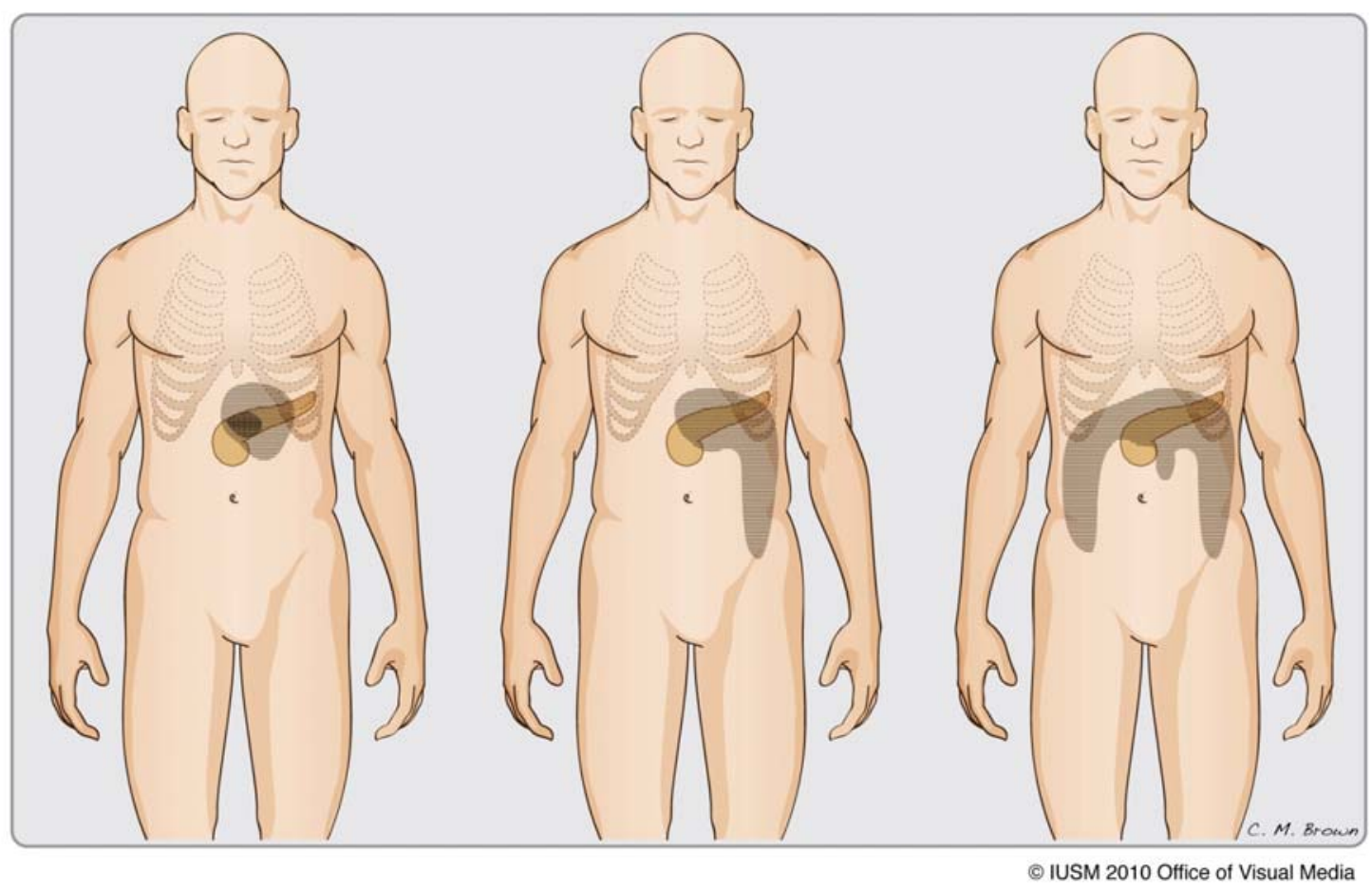


Figure 3:

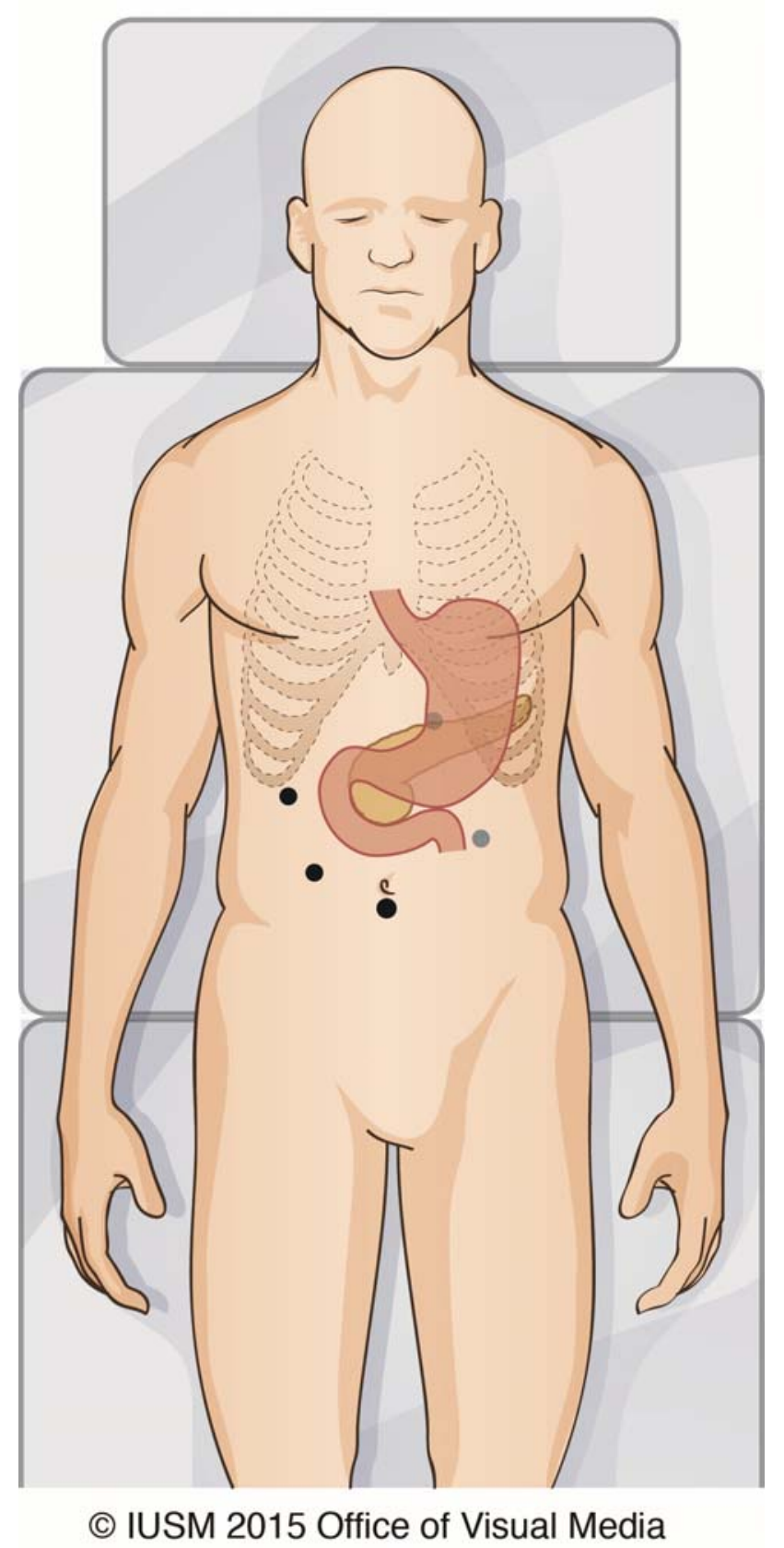


Figure 4:

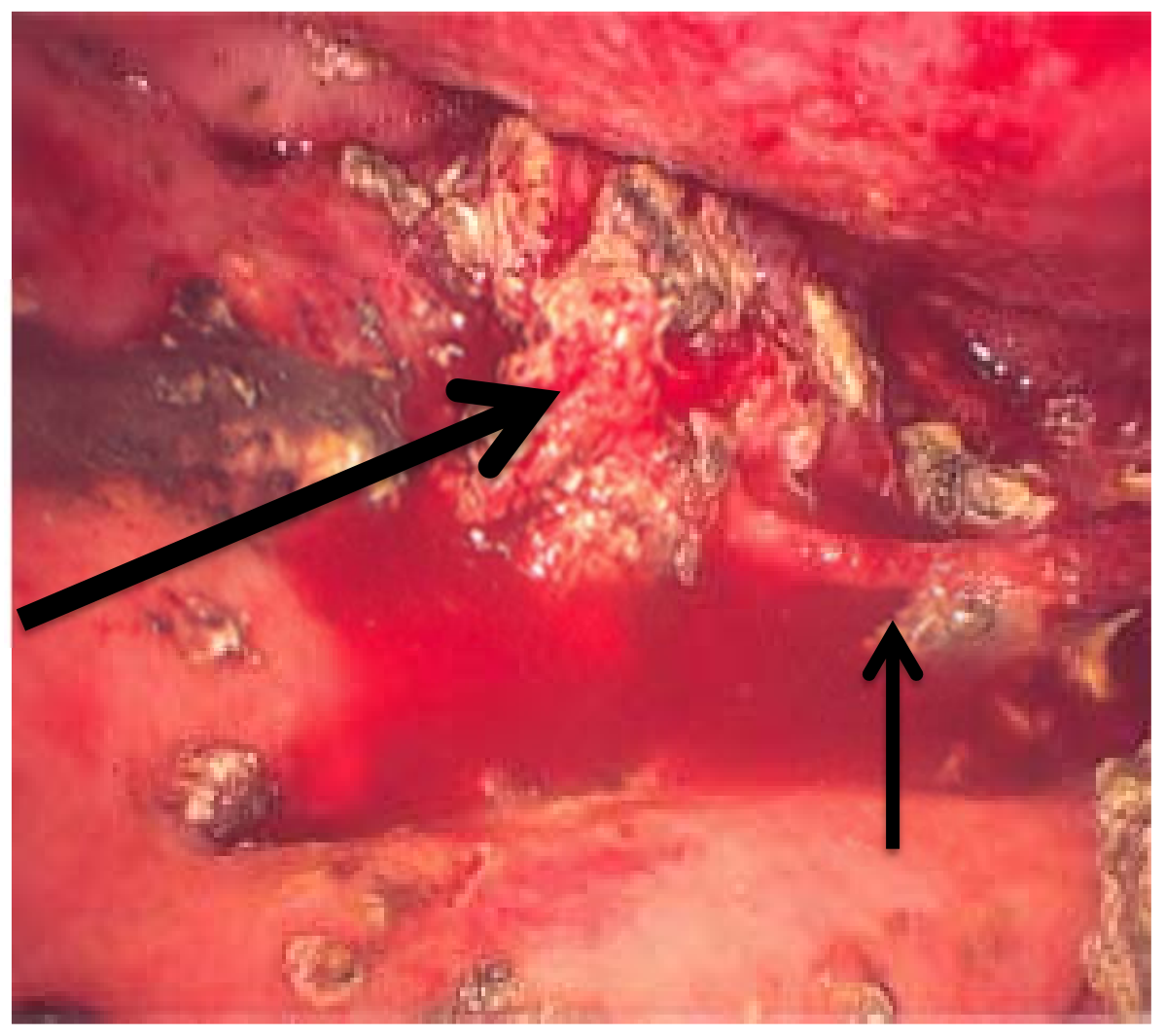

Figure 5: 


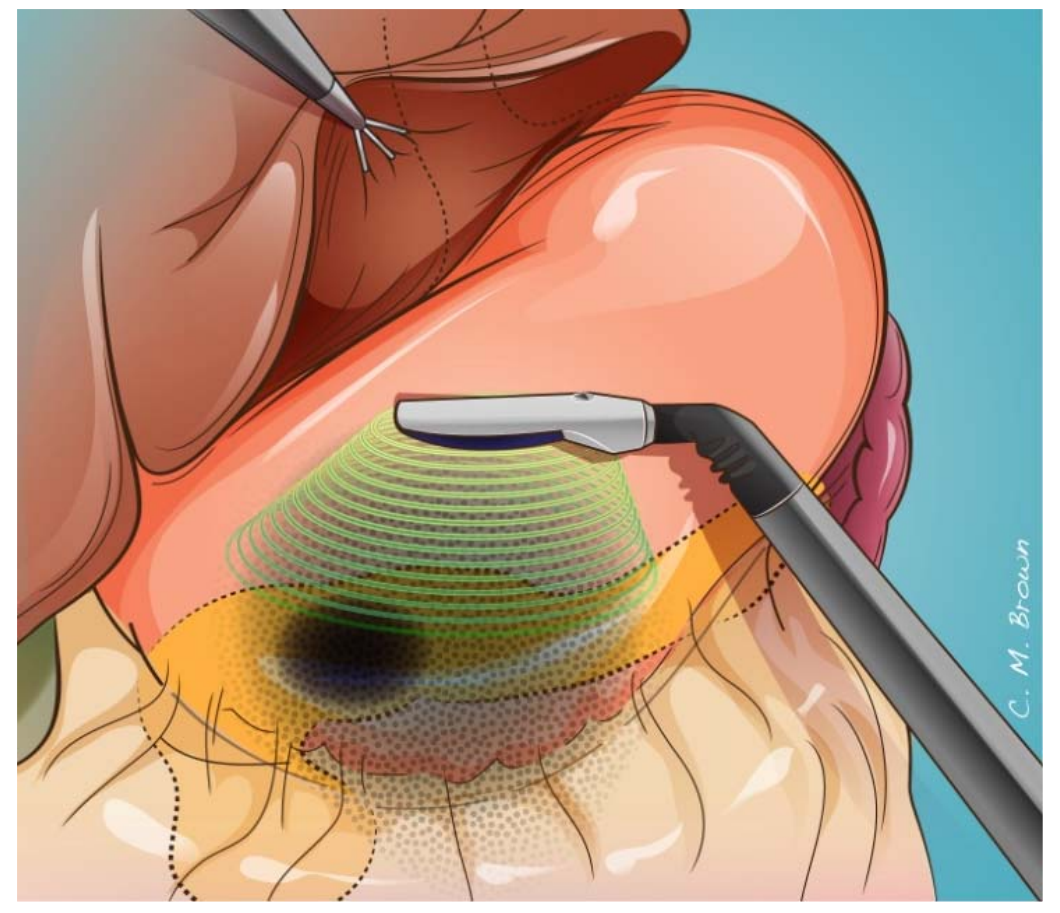

Figure 6:

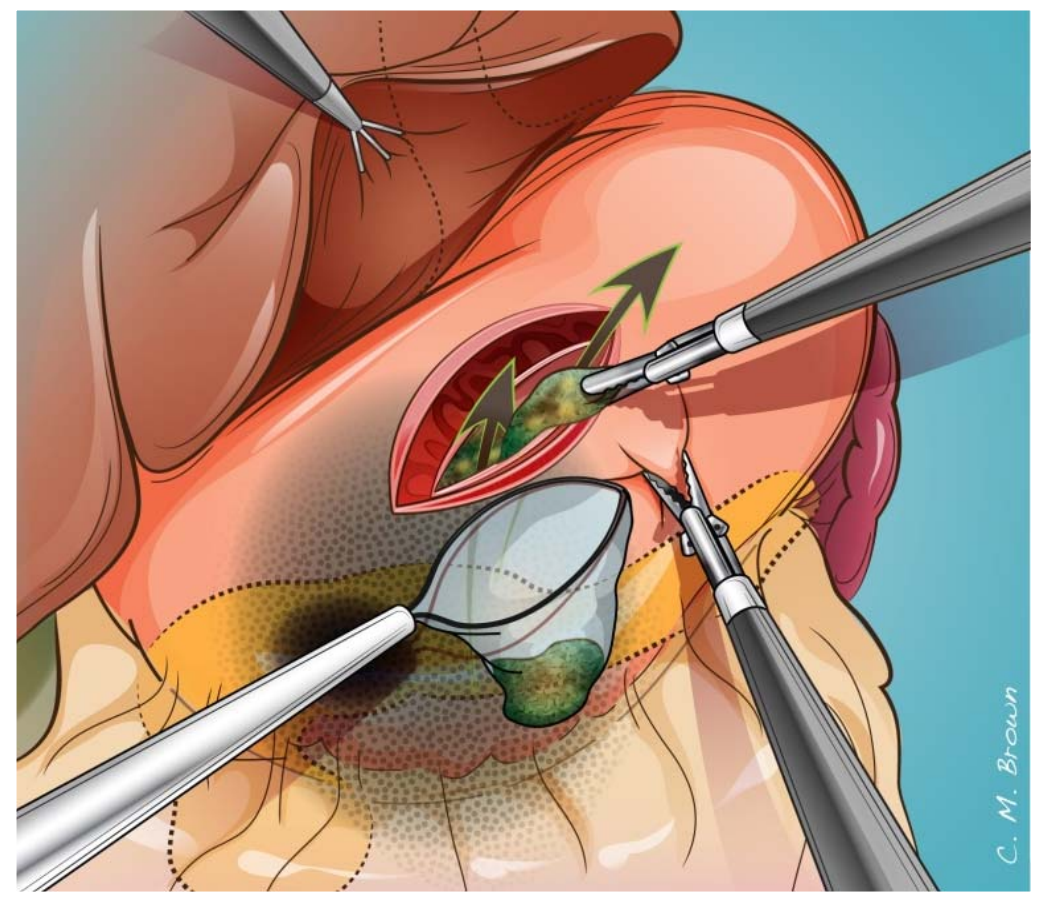

Figure 7: 
Zyromski Page 17

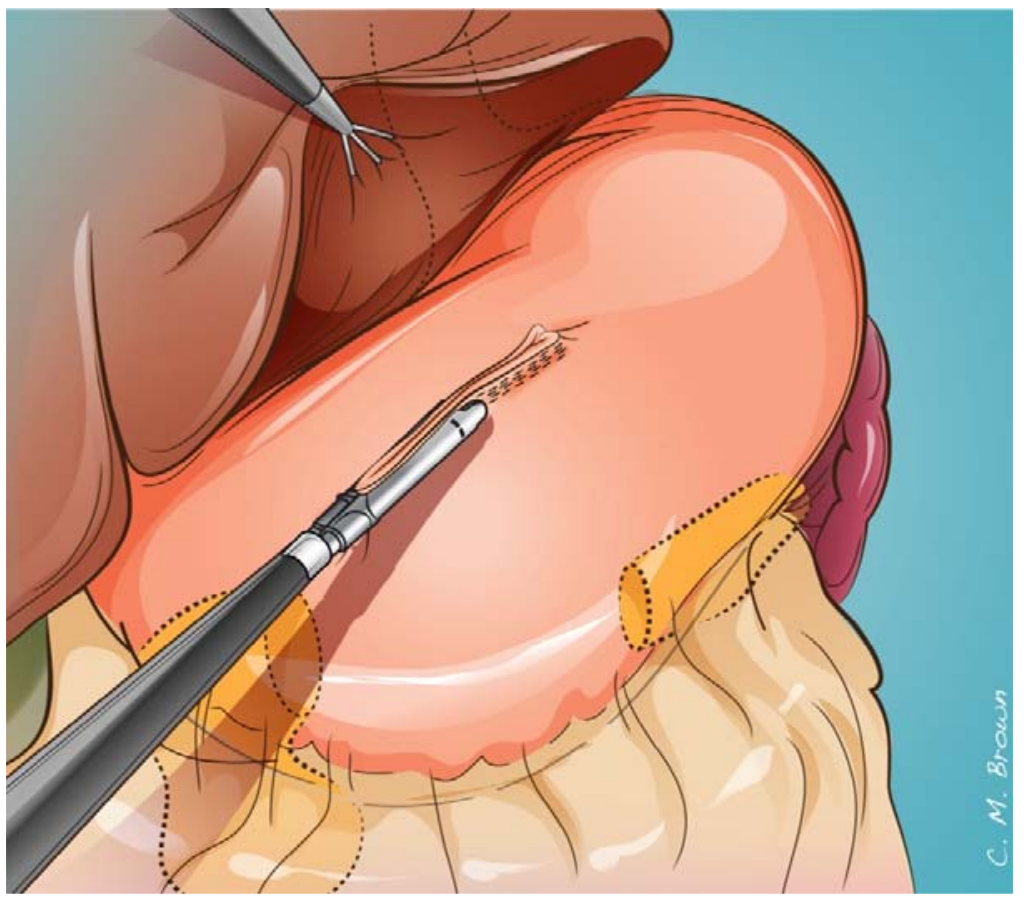

